\title{
Staphylococcal infection of the odontoid peg
}

\author{
Sean Keogh and Alan Crockard
}

The Department of Surgical Neurology, The National Hospital for Neurology and Neurosurgery, Maida Vale, London W9 1TL, UK

\begin{abstract}
Summary: An epidural abscess secondary to osteomyelitis of the odontoid peg produced a sudden left-sided weakness. Appropriate antibiotics, transoral evacuation of the pus and subsequent posterior atlanto-axial fusion were associated with a full recovery.
\end{abstract}

\section{Introduction}

Pyogenic infection involving the odontoid peg is particularly rare. In 1896 Makins and $\mathrm{Abbott}^{1}$ reported 2 cases of odontoid osteomyelitis and there have been only a handful of cases since. ${ }^{2-4}$

Atlanto-axial subluxation secondary to infective destruction of the transverse ligament of the atlas was first described by Bell ${ }^{5}$ in 1830 , commenting upon an eroding syphilitic posterior pharyngeal wall ulcer. In current medical practice, the most common association with such ligamentous destruction is parapharyngeal $\beta$-haemolytic streptococcal abscess chiefly in children. There are only occasional reports of infection-related atlanto-axial subluxation in adults..$^{6-8}$

The following case report combines both staphylococcal osteomyelitis of the odontoid peg with atlanto-axial subluxation. The movement of $\mathrm{Cl}$ on C2 resulted from secondary transverse ligament destruction associated with a local epidural abscess.

\section{Case report}

A 41 year old Caucasian was admitted to a London hospital with a 5 week history of gradually increasing neck pain radiating to his occiput. $\mathrm{He}$ also complained of general malaise, fever and weight loss of about $15 \mathrm{~kg}$. He admitted to intravenous heroin abuse, the last injection being 3 weeks before onset of symptoms. More recently he had developed increasing difficulty in swallowing due to a sore throat. At this time he had no neurological deficits

Correspondence: A. Crockard, F.R.C.S.

Accepted: 25 June 1991 but did have a swinging pyrexia. There was no history of tuberculosis, rheumatoid arthritis, diabetes or trauma to his neck.

Plain cervical films showed a $5 \mathrm{~mm}$ anterior shift of the atlas on the axis, progressing to $11 \mathrm{~mm}$ over several days and a soft tissue swelling anterior to the axis (Figure 1). Blood was taken for culture and he was started empirically on intravenous flucloxacillin and fusidic acid. Cultures subsequently grew Staphylococcus aureus which was sensitive to these antibiotics. Over several days his temperature settled.

Nine days post-admission he developed, over a matter of hours, a left-sided hemiparesis and spinothalamic diminution on the contralateral side from C4 distally. Emergency computed myelotomogram revealed a marked compression of the spinal cord at $\mathrm{Cl}-2$ by a non-encapsulated soft tissue mass surrounding and eroding the odontoid peg (Figure 2). The odontoid peg had subluxed to the left within the ring of the atlas indicating rupture of the transverse ligament (Figure 3). A provisional diagnosis of epidural abscess was made and the patient transferred to this hospital for surgery in skull traction.

On examination he was pyrexial $\left(38.1^{\circ} \mathrm{C}\right)$, with a pulse of 80 beats/minute and a blood pressure of $160 / 80 \mathrm{mmHg}$. Any neck manipulation induced pain. His pharynx was oedematous and mildly inflamed but without regional lymphadenopathy. No evidence of peripheral embolic phenomena could be found.

Positive neurological signs included an MRC grade IV hemiparesis of the left side and extensor plantar responses bilaterally. Spinothalamic sensory diminution at this time extended from T10 distally. Laboratory investigations revealed a white cell count of $17.9 \times 10^{9} / 1$ with $91 \%$ neutrophils. 


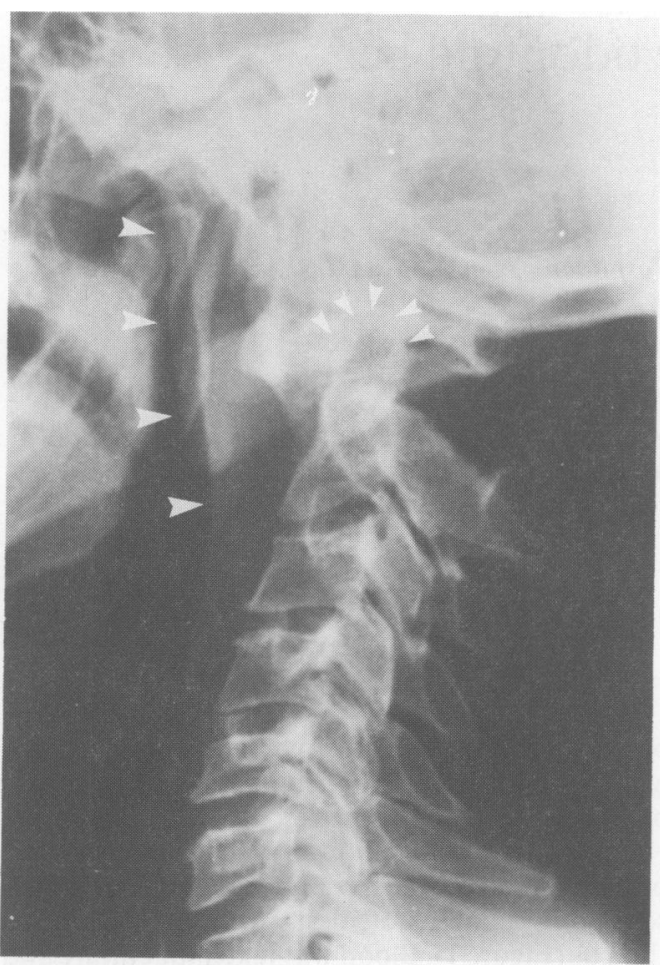

Figure 1 Lateral cervical radiograph, pre-operatively, showing the atlanto-axial subluxation and the soft tissue retropharyngeal swelling.

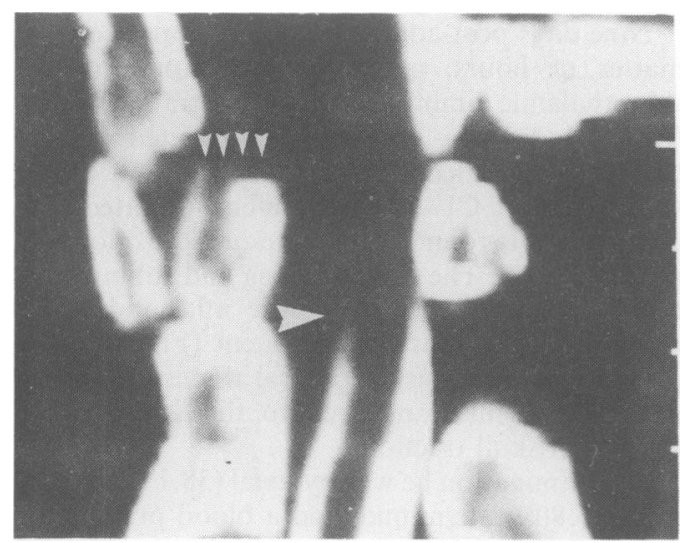

Figure 2 The sagittal reconstruction of the computed myelotomogram revealing erosion of the odontoid peg and a complete 'block' to the passage of contrast due to the epidural abscess.

Immunometric assay for anti-human immunodeficiency virus (HIV) 1 and 2 and screening for viral hepatitis proved negative. Random blood glucose was within normal limits.

The patient underwent emergency transoral

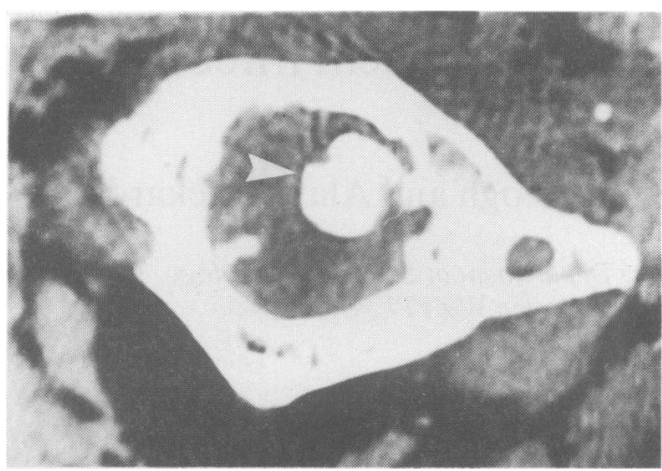

Figure 3 A coronal CT myelographic section revealing the displacement of the odontoid peg associated with a ruptured transverse ligament.

evacuation of extradural pus at $\mathrm{Cl}-2$ and excision of the eroded infected odontoid peg. He remained in skull traction post-operatively and over several days his hemiparesis resolved. Ten days later he underwent a posterior Brook's fusion using an iliac crest graft and sublaminar wire to stabilise his atlanto-axial joint (Figure 4). Histological examination of the excised odontoid peg confirmed the presence of clusters of Gram-positive cocci, with small foci of infarction. There was no evidence of tuberculosis. He made an uneventful and completê recovery and at 3 months was shown radiographic ally to have a sound atlanto-axial fusion.

\section{Discussion}

In osteomyelitis of any part of the spine clinical features can be subtle and varied, thus delaying diagnosis. This may be disastrous in infection involving the upper cervical spine, as reports suggest the more cephalad the disease process, the more likely paralysis is to develop. ${ }^{9}$ This paralysis may be irreversible. Late clinical diagnosis is compounded by the fact that the osseous changes of osteomyelitis may not be evident on plain X-ray for several weeks or months following infection and widening of the pre-vertebral soft tissue is easily missed, especially by the inexperienced. Myelography and computerized tomography are invaluable, though magnetic resonance imaging may now be the investigation of choice for the early recognition of dens axis osteomyelitis. ${ }^{10}$

The source of infection in this case was unclear. At transoral decompression the pharynx was oedematous and mildly inflamed. This may have been the bacterial origin. Grisel ${ }^{11}$ suggested that hyperaemia secondary to peripharyngeal inflammation could result in a lax transverse ligament with subsequent atlanto-axial subluxation. The pharyn- 


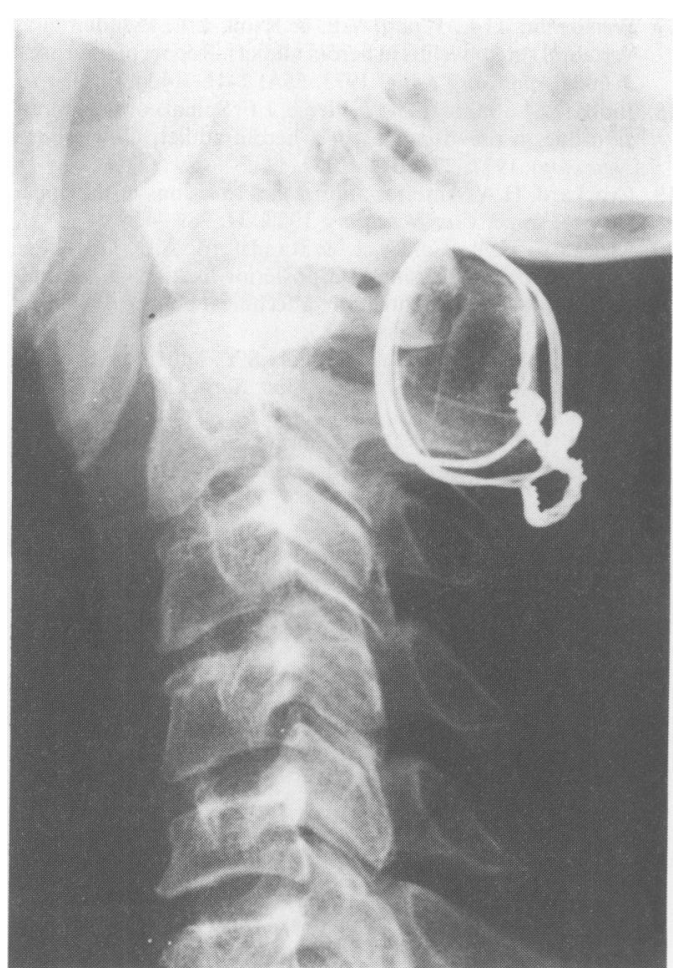

Figure 4 Lateral cervical radiograph, post-operatively, to demonstrate the normal alignment, and the posterior sublaminar wire and bone fusion.

govertebral venous system described by Parke $e t$ al. ${ }^{12}$ may provide a haematogenous route for the movement of septic material to the upper cervical structures.

Alternatively, septic emboli originating at a distant site may have caused the abscess formation, perhaps introduced by a non-sterile intravenous narcotic injection. There are several reports of osteomyelitis secondary to intravenous drug abuse. ${ }^{13-17}$ Infecting organisms tend to reflect the spectrum of pathogens affecting addicts, such as staphylococci, Gram-negative rods and yeasts. ${ }^{13}$

\section{References}

1. Makins, G.H. \& Abbott, F.C. On acute primary osteomyelitis of the vertebrae. Ann Surg 1896, 23: 510-539.

2. Frank, T.J.F. Osteomyelitis of the odontoid process of the axis. Med J Aust 1944, 1: 198-201.

3. Leach, R.E., Goldstein, H.H. \& Young, D. Osteomyelitis of the odontoid process. J Bone Joint Surg (Am) 1967, 49A: 369-371.

4. Rimalovski, A.B. \& Avonson, S.M. Abscess of medulla oblongata associated with osteomyelitis of odontoid process. Case report. J Neurosurg 1968, 29: 97-101.

5. Bell, C. The nervous system of the human body. (2nd edn.) Longman, London, 1830, p. 403.
Although our patient used sterile needles, the quality of the injected heroin was thought to be poor and the syringes non-sterile. Some reports have suggested that neurological deficits in vertebral osteomyelitis are in fact rare if associated with intravenous drug abuse. ${ }^{15-18}$ This may be related to the low age of the population involved (youth appears to be protective), the organism involved (often Pseudomonas aeruginosa rather than staphylococci) and the infrequent coexistence of systemic disease. If osteomyelitis is suspected in a drug abuser the usual empirical antibiotic treatment may not cover the wide variety of organisms in this population, and early surgical exploration may be appropriate.

It became clear in this case that with the development of paralysis and instability of the atlantoaxial joint, the epidural abscess required urgent evacuation and the cervical spine subsequent stabilization. As the purulent material was located anterior to the dens, the logical route for drainage was transorally. We have found this approach to be safe for a variety of pathology. ${ }^{19,20}$ The same route has been used for similarly situated tuberculous abscesses with success, both by this unit and elsewhere. ${ }^{21,22}$ Some authors advocate stabilization of the neck post-operatively in a halo brace for 3 months with $\mathrm{Cl}-2$ wiring and fusion if this conservative treatment fails. ${ }^{6}$ We prefer fusion as a first choice if practical. Posterior fusion may be performed at the time of transoral decompression and odontoidectomy, ${ }^{20}$ but considering the initial presence of pus we waited for 10 days and continued high dose antibiotics. Bone fusion and wound healing were unremarkable.

\section{Acknowledgements}

The authors are grateful to Dr J. Pringle, Consultant Pathologist, Royal National Orthopaedic Hospital, Stanmore for her help with this case and to M. Green for the preparation of this manuscript. The study was supported in part by a generous donation from Lex Brookland.

6. Clarke, W.C., Coscia, M., Acker, J.D., Wainscott, K. \& Robertson, J.T. Infection-related spontaneous atlanto-axial dislocation in an adult. $J$ Neurosurg 1988, 69: 455-458.

7. Swanberg, $H$. Anterior dislocation of the atlas following tonsillectomy. JAMA 1919, 72: 107-108.

8. Wilson, M.J., Michele, A.A. \& Jacobson, E.W. Spontaneous dislocation of the atlanto-axial articulation, including a report of a case with quadriplegia. J Bone Joint Surg 1940, B22: 698-707.

9. Eismont, F.J., Bohlman, H.H., Soni, P.L., Goldberg, V.M. \& Freehafer, A.A. Pyogenic and fungal vertebral osteomyelitis with paralysis. J Bone Joint Surg (Am) 1983, 65A: 19-29. 
10. Fischer, U. \& Vosshenrich, R. Osteomyelitis ('spinal infection', spondylitis, spondylodiszitis) des dens axis. Röntgenblatter 1990, 43: 54-57.

11. Grisel, P. Enucléation de l'atlas et torticolis naso-pharyngien. Presse Méd 1930, 38: 50-53.

12. Parke, W.W., Rothman, R.H. \& Brown, M.D. The pharyngovertebral veins: an anatomical rationale for Grisel's Syndrome. J Bone Joint Surg (Am) 1984, 66A: 568-574.

13. Holzman, R.S. \& Bishko, F. Osteomyelitis in heroin addicts. Ann Intern Med 1971, 75: 693-696.

14. Kido, D., Bryan, D. \& Halpern, M. Haematogenous osteomyelitis in drug addicts. Ann $J$ Roentgenol 1973, 118: 356-363.

15. Lewis, R., Gorbach, S. \& Altner, P. Spinal pseudomonas chondroosteomyelitis in heroin users. $N$ Engl J Med 1972 , 286: 1303.

16. Messer, H.D. \& Litvinoff, J. Pyogenic cervical osteomyelitis. Chrondro-osteomyelitis of the cervical spine frequently associated with parenteral drug use. Arch Neurol 1976, 33: 571-576.
17. Weisseman, G.J., Wood, V.E. \& Kroll, L.L. Pseudomonas vertebral osteomyelitis in heroin addicts. Report of five cases. $J$ Bone Joint Surg (Am) 1973, 55A: 1416-1424.

18. Jabbari, M., Bahman, K. \& Pierce, J.F. Spinal cord compression due to pseudomonas in a heroin addict. Case report. Neurology 1977, 27: 1034-1037.

19. Crockard, H.A. Anterior approaches to lesions of the upper cervical spine. Clin Neurosurg 1988, 34: 389-416.

20. Crockard, H.A., Calder, I. \& Randsford, A.O. One stage transoral decompression and posterior fixation in rheumatoid atlanto-axial subluxation: a technical note. J Bone Joint Surg (Br) 1990, 72B: 682-685.

21. Fang, D., Leong, J.C.Y. \& Fang, H.S.Y. Tuberculosis of the upper cervical spine. J Bone Joint Surg (Br) 1983, 65B: 47-50.

22. Wang, L.X. Peroral focal debridement for treatment of tuberculosis of the atlas and axis. Chin J Orthop 1981, 1: 207-209. 